\title{
Real-Time Modeling of Distributed Plug-in Vehicles for V2G Transactions
}

\author{
Ganesh K. Venayagamoorthy, Senior Member, IEEE, Pinaki Mitra, Student Member, \\ IEEE, Keith Corzine, Senior Member, IEEE, and Chris Huston, Student Member, IEEE
}

\author{
Real-Time Power and Intelligent Systems Laboratory \\ Missouri University of Science and Technology, Rolla, MO 65409, USA \\ gkumar@ieee.org; http://rtpis.mst.edu
}

\begin{abstract}
A real-time model of a fleet of plug-in vehicles performing vehicle-to-grid (V2G) power transactions is presented in this paper. Two sets of four vehicles are connected to both the grid and to each other though a short transmission line. The real-time modeling is carried out on a Real-Time Digital Simulator (RTDS). This setup allows for two different case studies with either two distributed sets of four individual vehicles or two smart parking lots with several vehicles. Output power levels and charge/discharge times are scheduled intelligently in order to maximize profits from grid transactions based on one-day ahead electricity pricing. The effects of grid faults on V2G operations are presented.
\end{abstract}

Index Terms - computational intelligence, particle swarm optimization, plug-in vehicles, power transactions, real-time simulation, real-time digital simulator, smart parking lots, vehicle-to-grid (V2G)

\section{INTRODUCTION}

As increasing numbers of plug-in hybrid electric (PHEVs) and electric vehicles (EVs) enter the market, analysis tools are needed to determine the effects of adding large numbers of small power electronic devices to the grid. Many of these vehicles can also be adopted to participate in vehicle-to-grid (V2G) applications in the proposed smart grid framework which calls for bidirectional power flows between vehicles and the electric utility grid [1-2]. The plug-in vehicles can provide many grid services such as regulation or spinning reserves, or they could generate revenue by buying and selling power at different times according to variable price curves [3]. In order to have a significant effect on the grid in terms of power transactions, very large numbers of vehicles must be aggregated together. In all of these applications, large numbers of interacting power electronic devices are involved and these may cause possible interactions between the inverters and on the utility grid.

Vehicles providing regulation or spinning reserves coordinate their power flows with the utility to change grid conditions in some predetermined way. If vehicle owners try to buy and sell power according to varying prices, the utility must cope with large swings in power as groups of vehicles switch the direction of their power flows.

The supports from the National Science Foundation under the grant EFRI \# 0836017 is gratefully acknowledged by the authors for this work.
In addition to varying power flows, the utility does not know the exact number of vehicles participating at any given time making it difficult to forecast the necessary power demand. This type of charging is referred to as the 'random' or 'dump' charging/discharging scenario and is the worst case for the utility grid in terms of large fluctuations in load demand. 'Smart' charging requires intelligent scheduling.

An eight vehicle real-time simulation incorporating intelligent scheduling of power flows for profit maximization is presented in this paper. The system studied consists of two sets of four vehicles that simulate the V2G operation. In addition, a second case study looking at an aggregated model of several hundreds of vehicles is also examined for grid operations. The results show the varying power flows from each vehicle and the overall impact of large number of vehicles have on the grid. Effects of grid faults on $V 2 G$ operations are presented.

\section{DistRIBUTED SMARTPARKS WITH PlUG-IN VEHICLES}

The system studied in this paper consists of eight interconnected three-phase vehicles (inverters) tied to the utility distribution grid through a step-up transformer $(208 \mathrm{~V} / 22.9 \mathrm{kV})$ as shown in Fig. 1. The system is modeled on a real-time digital simulator (RTDS) platform [4]. In the current setup, four inverters are modeled in one RTDS rack. The two smart parking lots (SmartParks) are connected to each other through a $15 \mathrm{~km}$ transmission line (line parameters given in Table I). In a typical city, there will be several SmartParks distributed throughout the city in distances of one to few kilometers. Two case studies are considered in this paper. The first one is case where four vehicles are considered in each SmartPark. The second case study is one where are hundreds of vehicles are aggregated in each SmartPark.

The simulation of the vehicle inverters is carried out smaller time steps on the giga processor RTDS cards, the GPC cards. The GPC is a powerful computational unit which can be used for solving the overall network solution as well as auxiliary components. The GPC contains two IBM PowerPC 750GX RISC processors each operating at $1 \mathrm{GHz}$. In addition to the network solution and the simulation of standard components at $50 \mu$ s time step, the GPC card provides small time step $(<2 \mu \mathrm{s})$ simulations for voltage source converters (VSC). This increase in accuracy allows for better representation of the switching components in a realtime environment. The inverters are designed to operate at 
$208 \mathrm{~V}$ using a three phase two level topology and are controlled using a compensation controller [5]. For cases where each inverter represents a single vehicle, a $5 \mathrm{mH}$ inductor is used between the switching components and the transformer.

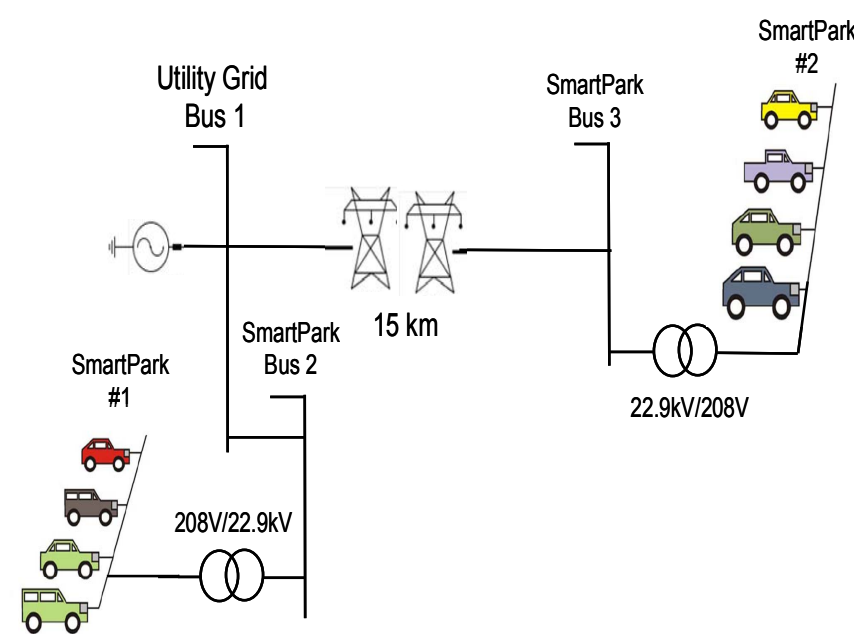

Fig. 1. Two SmartParks connected to the utility grid through a step-up transformer. In case of aggregated model, hundreds of vehicles are considered in each SmartPark.

TABLE I

TRANSMISSION LINE PARAMETERS

\begin{tabular}{|l|c|}
\hline Positive Sequence Series Resistance & $0.3933 \Omega / \mathrm{km}$ \\
\hline Positive Sequence Series Inductive Reactance & $1.0882 \Omega / \mathrm{km}$ \\
\hline Positive Sequence Shunt Capacitive Reactance & $0.1470 \mathrm{M} \Omega \mathrm{km}$ \\
\hline Zero Sequence Series Resistance & $1.2088 \Omega / \mathrm{km}$ \\
\hline Zero Sequence Series Inductive Reactance & $3.0022 \Omega / \mathrm{km}$ \\
\hline Zero Sequence Shunt Capacitive Reactance & $0.2132 \mathrm{M} \Omega \mathrm{km}$ \\
\hline Line Length & $15.0 \mathrm{~km}$ \\
\hline
\end{tabular}

\section{INTELLIGENT SMARTPARK SCHEDULING}

In order to test the multi-inverter system with a potential scenario, the power flows are scheduled by a binary particle swarm optimization (BPSO) algorithm [6]. The algorithm searches for near optimal times to buy and sell power in order to generate revenues for the vehicles participating in V2G operations. An optimal schedule results in the maximum possible profits earned. These profits are possible assuming that the vehicles have access to the real-time electricity price information which is known to vary throughout the day.

The BPSO algorithm used by Khanesar [7] modifies the classical BPSO algorithm [8] by adding a second velocity equation which separates the probabilities of changing a bit to zero and changing a bit to one. The velocities, $V_{i j}{ }^{0}$ and $V_{i j}{ }^{l}$, are updated according to equations in (1) to (6). Next either $V_{i j}{ }^{0}$ or $V_{i j}{ }^{l}$ are chosen by (7) and then normalized by (8). The particle position is updated by (9) where the normalized probability of flipping the current bit is compared to a uniform random number. The overall result of this method is that the probability of bits flipping is more directly tied to the values stored in the particle's best $\left(P_{\text {best }}\right)$ and the swarm best $\left(G_{\text {best }}\right)$. In standard PSO, the velocity gets closer to zero when the particle is close to the optimal solution. The problem arises when the sigmoid function is applied to the velocity in BPSO. A velocity of zero has a value of 0.5 after applying the sigmoid function which increases the probability bits are flipped.

$$
\begin{aligned}
& \text { If } P_{\text {best }}^{j}=1 \text { then } d_{i j, 1}{ }^{l}=c_{I} * r_{I} \text { and } d_{i j, l}=-c_{I} * r_{I} \\
& \text { If } P_{b e s t}=0 \text { then } d_{i j, 1}=c_{1} * r_{1} \text { and } d_{i j, 1}=-c_{1} * r_{1} \\
& \text { If } G_{b e s t}{ }^{j}=1 \text { then } d_{i j, 2}{ }^{l}=c_{1} * r_{1} \text { and } d_{i j, 2}=-c_{1} * r_{1} \\
& \text { If } G_{b e s t}{ }^{j}=0 \text { then } d_{i j, 2}{ }^{0}=c_{1} * r_{1} \text { and } d_{i j, 2}{ }^{1}=-c_{1} * r_{1} \\
& V_{i j}^{1}=w^{*} V_{i j}^{1}+d_{i j, 1}^{1}+d_{i j, 2}^{1} \\
& V_{i j}^{0}=w^{*} V_{i j}^{0}+d_{i j, 1}^{0}+d_{i j, 2}^{0} \\
& V_{i j}^{c}= \begin{cases}V_{i j}^{c}=V_{i j}^{1} & \text { if }, x_{i j}=0 \\
V_{i j}^{c}=V_{i j}^{0} & \text { if }, x_{i j}=1\end{cases} \\
& V_{i j}^{\prime}=\frac{1}{1+e^{-V_{i j}^{c}}} \\
& x_{i j}(t+1)= \begin{cases}\bar{x}_{i j}(t) & \text { if }, r_{i j}<V_{i j}^{\prime} \\
x_{i j}(t) & f, r_{i j}>V_{i j}^{\prime}\end{cases}
\end{aligned}
$$

In this application, two bits are used to describe the vehicle status. Using ' 11 ' and ' 00 ' as idle, ' 10 ' as sell, and ' 01 ' as buy, the vehicle is commanded to buy, sell, or stay idle. A buy operation consists of buying at a power transfer rate to charge to the maximum for that hour (or over the next few buying hours) or a limited maximum power transfer rate. And, likewise with the sell operation commands. The vehicle can sell at its maximum possible power for that hour or over a few hours without depleting the battery below a predefined level.

\section{Real-Time Simulation of V2G TRANSACtions}

The parameters used for each vehicle in the first case study are shown in Table II. The arrival and departure times define which hour(s) of the day a vehicle is committed to participate in V2G operations. For example, hour 1 represents the time between 12:00AM and 1:00AM while hour 24 represents 11:00PM to 12:00AM. The battery size used is taken to be equal for all vehicles in this study and is $25 \mathrm{kWh}$. The maximum depth of discharge is the lowest allowed battery level when discharging. A vehicle can arrive with a battery depleted further than this limit, but the V2G operation is not allowed to discharge the battery when the battery is below this level. Lastly the state of charge of battery on arrival parameter is the battery level when a vehicle enters into V2G operation, and the overall efficiency accounts for the battery and inverter efficiencies.

The electricity price used for determining revenue is taken from the California Independent System Operators (CAISO) website for August 7, 2008. Figure 2 shows the 
hourly market clearing price in dollars per MWh. Prices at each hour are taken from the graph and used by BPSO scheduling algorithm.

TABLE II

VEHICLE PARAMETERS

\begin{tabular}{|l|c|c|c|c|c|c|c|c|}
\hline Vehicle \# & $\mathbf{\# 1}$ & $\mathbf{\# 2}$ & $\mathbf{\# 3}$ & $\mathbf{\# 4}$ & $\mathbf{\# 5}$ & $\mathbf{\# 6}$ & $\mathbf{\# 7}$ & $\mathbf{\# 8}$ \\
\hline Arrival Hour & 13 & 7 & 2 & 1 & 13 & 7 & 2 & 1 \\
\hline Departure Hour & 18 & 21 & 22 & 15 & 18 & 21 & 22 & 15 \\
\hline Battery Size (kWh) & 25 & 25 & 25 & 25 & 25 & 25 & 25 & 25 \\
\hline $\begin{array}{l}\text { Maximum Depth of } \\
\text { Battery Discharge } \\
\text { (\%) }\end{array}$ & 60 & 80 & 60 & 80 & 60 & 80 & 60 & 80 \\
\hline $\begin{array}{l}\text { State of Charge of } \\
\text { Battery on Arrival } \\
\text { (\%) }\end{array}$ & 65 & 49 & 70 & 98 & 84 & 52 & 75 & 90 \\
\hline $\begin{array}{l}\text { Overall Battery and } \\
\text { Inverter Efficiency } \\
(\%)\end{array}$ & 85 & 90 & 90 & 84 & 87 & 92 & 80 & 86 \\
\hline
\end{tabular}

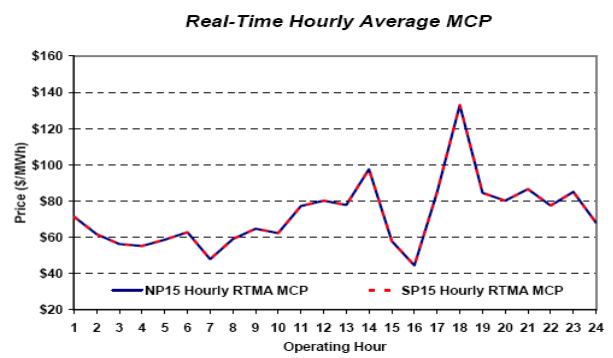

Fig. 2. CAISO Market Clearing Prices on August 8, 2008

The scheduling found by the BPSO algorithm shows the appropriate times for the respective vehicles to participate in the V2G operations. Table III shows the schedule for each vehicle throughout the period of one day. Buy and sell operations are denoted by '-' and '+' signs respectively, while idle is simply a ' 0 '. In other words, '- 'means charging operation and ' + ' means discharging operation by a vehicle from and to the grid respectively. Reference power transfer levels are determined for the parameters specified by Table II for each vehicle.

Fig. 3 shows the individual vehicle scheduled power transfers based on schedule generated on August 7, 2008 price information. Vehicles \#1 to \#4 are parked in SmartPark $\# 1$ and vehicles \#5 to \#8 are parked in SmartPark \#2. Fig. 4 shows the cumulative power V2G transactions on the same day from the two SmartParks. The V2G transactions can be analyzed by comparing the buy (charge) and sell (discharge) times to the price graph in Fig. 2. Buy and sell operations coordinate with high and low prices to make higher profits. Hours 7 and 16 are the best times to charge from the grid, and hours 14 and 18 are the best times to discharge to the grid. Since the times the vehicles are available are staggered throughout the day and have different efficiencies, different buy and sell strategies are evolved for each vehicle. All vehicles but vehicles \#2 and \#6 found that many buy and sell cycles resulted in the highest revenues while vehicle $\# 2$ and \#6 found that simply buying at hour 16 was most profitable. Vehicles \#4 arrives with $98 \%$ full battery level and the minimum state of charge for departure is $80 \%$, therefore the reference power transfer levels are small compared to other vehicles in the SmartParks, as seen in Fig. 3.
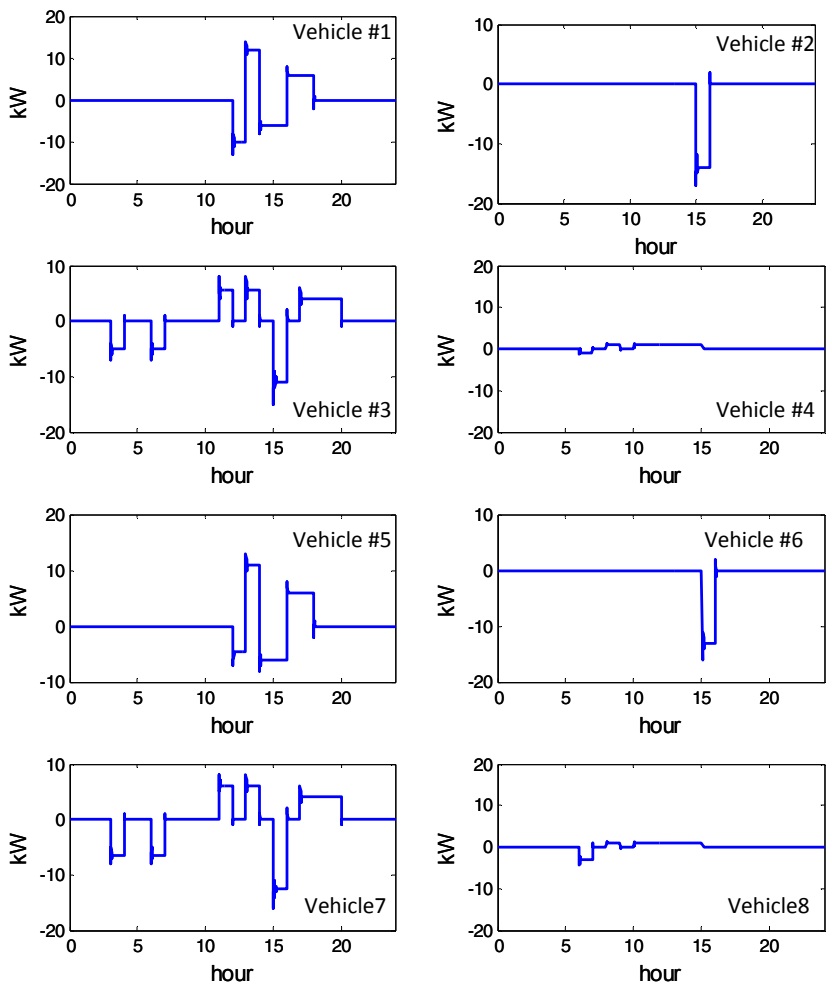

Fig. 3. Vehicle power transactions in their respective SmartParks based on August 7, 2008 electricity price information

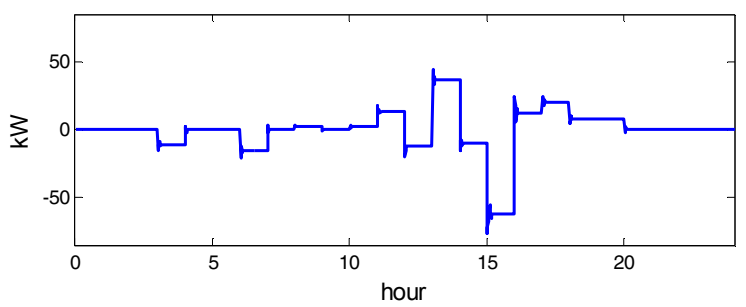

Fig. 4. Cumulative V2G power transactions based on August 7, 2008 electricity price information

Fig. 5 shows vehicle \#1's charging operation. It can be seen that the inverter follows closely the charging power level command. The overshoot is seen to be $20 \%$ of the reference power level but it gets damped out within 0.5 seconds of the start of the charging operation. Of course, some optimal tuning is needed for different vehicles' inverter controls to participate in small and large power transactions. 
TABLE III

VeHicle SCHEdules AND POWER TRANSFER REFERENCE LEVELS

\begin{tabular}{|c|c|c|c|c|c|c|c|c|c|c|c|c|c|c|c|c|c|c|}
\hline \multirow[b]{2}{*}{$\begin{array}{c}\text { Vehicle } \\
\# \\
\end{array}$} & \multicolumn{18}{|c|}{ Reference Power Transfer Levels in kW for the Respective Vehicles and Hour(s) } \\
\hline & $1-3$ & 4 & 5-6 & 7 & 8 & 9 & 10 & 11 & 12 & 13 & 14 & 15 & 16 & 17 & 18 & 19 & 20 & 21-24 \\
\hline 1 & 0 & 0 & 0 & 0 & 0 & 0 & 0 & 0 & 0 & -10 & +12 & -6 & -6 & +6 & +6 & 0 & 0 & 0 \\
\hline 2 & 0 & 0 & 0 & 0 & 0 & 0 & 0 & 0 & 0 & 0 & 0 & 0 & -14 & 0 & 0 & 0 & 0 & 0 \\
\hline 3 & 0 & -5 & 0 & \begin{tabular}{l|}
-5 \\
\end{tabular} & 0 & 0 & 0 & 0 & +5.5 & 0 & +5.5 & 0 & -11 & 0 & +4 & +4 & +4 & 0 \\
\hline 4 & 0 & 0 & 0 & -0.6 & 0 & +1 & 0 & +1 & +1 & +1 & +1 & +1 & 0 & 0 & 0 & 0 & 0 & 0 \\
\hline 5 & 0 & 0 & 0 & 0 & 0 & 0 & 0 & 0 & 0 & -4.6 & +11 & -6 & -6 & +6 & +6 & 0 & 0 & 0 \\
\hline 6 & 0 & 0 & 0 & 0 & 0 & 0 & 0 & 0 & 0 & 0 & 0 & 0 & -13 & 0 & 0 & 0 & 0 & 0 \\
\hline 7 & 0 & -6.5 & 0 & -6.5 & 0 & 0 & 0 & 0 & +6.2 & 0 & +6.2 & 0 & -12.5 & 0 & +4 & +4 & +4 & 0 \\
\hline 8 & 0 & 0 & 0 & -3 & 0 & +1 & 0 & +1 & +1 & +1 & +1 & +1 & 0 & 0 & 0 & 0 & 0 & 0 \\
\hline
\end{tabular}

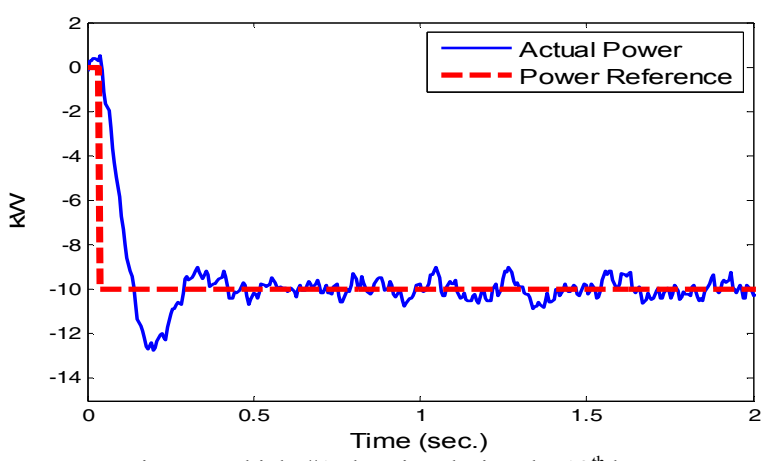

Fig. 5. Vehicle $\# 1$ charging during the $13^{\text {th }}$ hour

In order to investigate the effects of grid faults on vehicles of the SmartParks, a 10 cycle three phase short circuit is placed first on utility grid (Bus 1 in Fig. 1) and then on Bus 3 (SmartPark \#2 bus in Fig. 1). The fault is placed during the $14^{\text {th }}$ hour when six of the eight vehicles are selling power to the grid.

Fig. 6 (a) shows the plots of power flows between the vehicles and the SmartPark buses for a short circuit placed on the utility grid. Vehicles \#1 to \#4 parked in SmartPark \#1 experience large surge power magnitude, in both directions (power flowing from vehicle to the grid and vice-versa) whereas vehicles \#5 to \#8 parked in SmartPark \#2, $15 \mathrm{kms}$ away from the fault experience lesser surge power magnitudes. Fig. 6 (b) shows the plots of power flows between the vehicles and the SmartPark buses for a short circuit placed on the Bus 3 (SmartPark \#2 bus). In this case, vehicles \#5 to \#8 experience the larger power surges and vehicles \#1 to \#4 experience smaller power surges.

These results pose the need for better control and protection to the SmartParks. The rapid changes in power flows with large magnitudes are may be detrimental to the health of the batteries and the inverters in the vehicles.
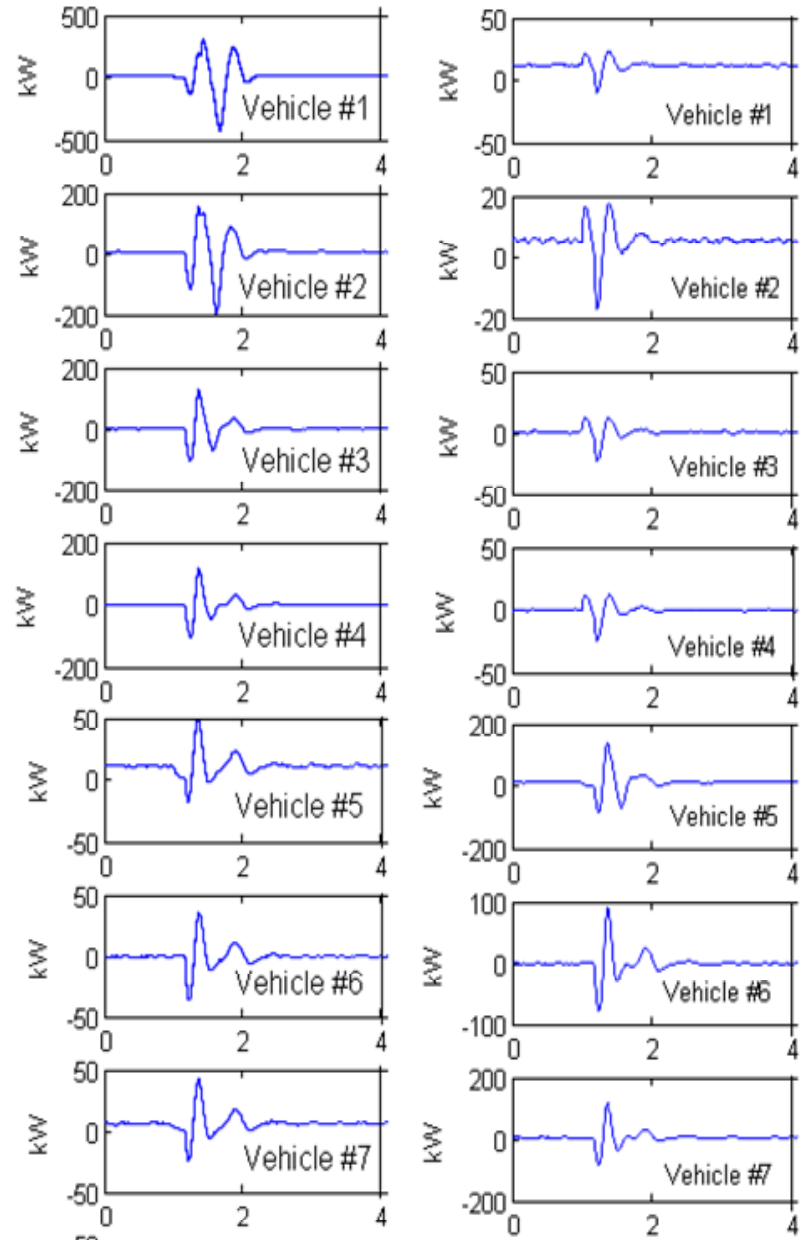

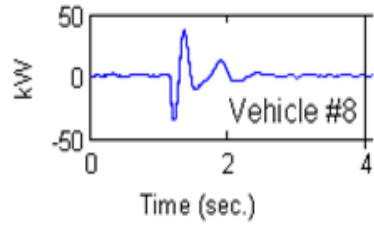

(a)

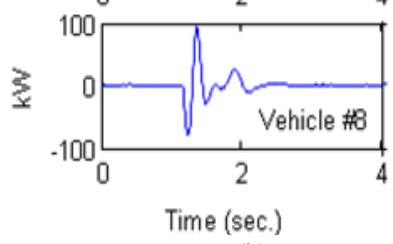

(b)
Fig. 6. 10 cycle three phase short circuit during the $14^{\text {th }}$ hour of V2G transactions (a) fault location is on the utility grid (Bus 1) (b) fault location on the SmartPark bus (Bus 3) 
TABLE IV

AgGREGATED VEHICLE SCHEDULES

\begin{tabular}{|c|c|c|c|c|c|c|c|c|c|c|c|c|c|c|c|c|c|c|c|c|c|c|c|c|}
\hline & 1 & 2 & 3 & 4 & 5 & 6 & 7 & 8 & 9 & 10 & 11 & 12 & 13 & 14 & 15 & 16 & 17 & 18 & 19 & 20 & 21 & 22 & 23 & 24 \\
\hline Inverter 1 & & & & & & & & & & & & & B & $\mathbf{S}$ & B & B & $\mathbf{S}$ & $\mathbf{S}$ & & & & & & \\
\hline Inverter 2 & & & & & & & - & - & - & - & - & - & - & - & - & B & - & - & - & - & - & & & \\
\hline Inverter 4 & - & - & - & - & - & - & B & - & $\mathbf{S}$ & - & $\mathbf{S}$ & $\mathbf{S}$ & $\mathbf{S}$ & $\mathbf{S}$ & $\mathbf{S}$ & & & & & & & & & \\
\hline
\end{tabular}

A second case study was carried out to investigate the integration of large number of vehicles to the utility grid where bidirectional power flows are in the MW range. Fig. 7 shows SmartPark \#1 configured into four sub-Smartparks, each with about 1000 vehicles, carrying out power transactions as an aggregator. The four invertors each aggregate about 1000 plug-in vehicles. The schedule generated by BPSO given in Table IV is based on the electricity price information of August 7, 2008 obtained CAISO.
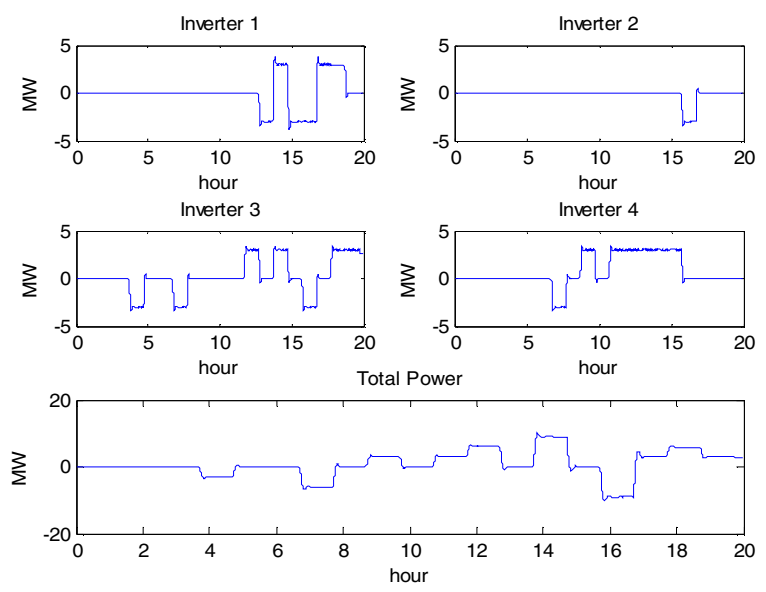

Fig. 7. Aggregated vehicle power transactions based on August 7, 2008 electricity price information

\section{CONCLUSION}

A real-time RTDS model of a fleet of plug-in vehicles performing vehicle-to-grid (V2G) power transactions has been presented in this paper. Two SmartPark models, each consisting of four vehicles, are connected to both the grid and to each other though a short transmission line. The reference power transfer levels and charge/discharge times are scheduled intelligently in order to maximize revenues from grid transactions based on one-day ahead electricity pricing. It is observed that grid faults can be detrimental to the vehicles during V2G transactions. Advanced control and protection is needed to avoid any adverse effects caused from the large bidirectional power surges to the batteries and the inverters of individual plug-in vehicles. Such advanced control and protection methods may be incorporated into the SmartParks at higher power levels and into the individual vehicles at smaller power levels. All V2G plug-in stations, be it public or private (including home garages) require intelligent control and protection devices for successful V2G operations in the proposed smart grid framework. Future work will investigate into such advanced control and protection methods.

\section{REFERENCES}

[1] W. Kempton, J. Tomić, "Vehicle-to-grid power fundamentals: Calculating capacity and net revenue," Journal of Power Sources, Volume 166 Issue 2, 15 April 2007, Pages 549-566

[2] AC Propulsion, "AC-150 EV power system", http://www.acpropulsion.com/tzero/AC150_Gen2_specs.pdf

[3] Hutson, Chris M., Venayagamoorthy, Ganesh K., Corzine, Keith A. "Intelligent Scheduling of Hybrid and Electric Vehicle Storage Capacity in a Parking Lot for Profit Maximization in Grid Power Transactions" IEEE Energy 2030, 2008, 17-18 November 2008.

[4] P. Forsyth, T. Maguire, R. Kuffel, "Real time digital simulation for control and protection system testing," IEEE 35th Annual Power Electronics Specialists Conference, vol. 1, pp. 329 - 335, 2004.

[5] P.C. Krause, O. Wasynczuk and S.D. Sudhoff, Analysis of Electric Machinery and Drive Systems, IEEE Press, 2002.

[6] Y. del Valle, G. K. Venayagamoorthy, S. Mohagheghi, J.-C. Hernandez, and R. G. Harley, "Particle swarm optimization: Basic concepts, variants and applications in Power Systems," IEEE Trans. Evolutionary Computation, vol. 12, no. 2, pp. 171-195, Apr 2008.

[7] Khanesar, M.A.; Teshnehlab, M.; Shoorehdeli, M.A.; "A novel binary particle swarm optimization," Mediterranean Conference on Control \& Automation. 27-29 June 2007 Page(s):1-6

[8] J. Kennedy and R.C. Eberhart, "A discrete binary version of the particle swarm algorithm," IEEE Conference on Systems, Man and Cybernetics, pages 4104-4108, Orlando FL, 1997. 